\title{
Strengthening the Study on the Behavior and Transformation of Medium-Chain Chlorinated Paraffins in the Environment
}

\author{
Yawei Wang, ${ }^{\dagger} \dagger, \downarrow, \S \odot$ Wei Gao, ${ }^{\dagger, \S}$ and Guibin Jiang
}

${ }^{\dagger}$ State Key Laboratory of Environmental Chemistry and Ecotoxicology, Research Center for Eco-Environmental Sciences, Chinese Academy of Sciences, 18 Shuangqing Road, Haidian District, Beijing 100085, China

${ }^{\ddagger}$ Institute of Environment and Health, Jianghan University, Wuhan 430056, China

${ }^{\S}$ University of Chinese Academy of science, Beijing 100049, China

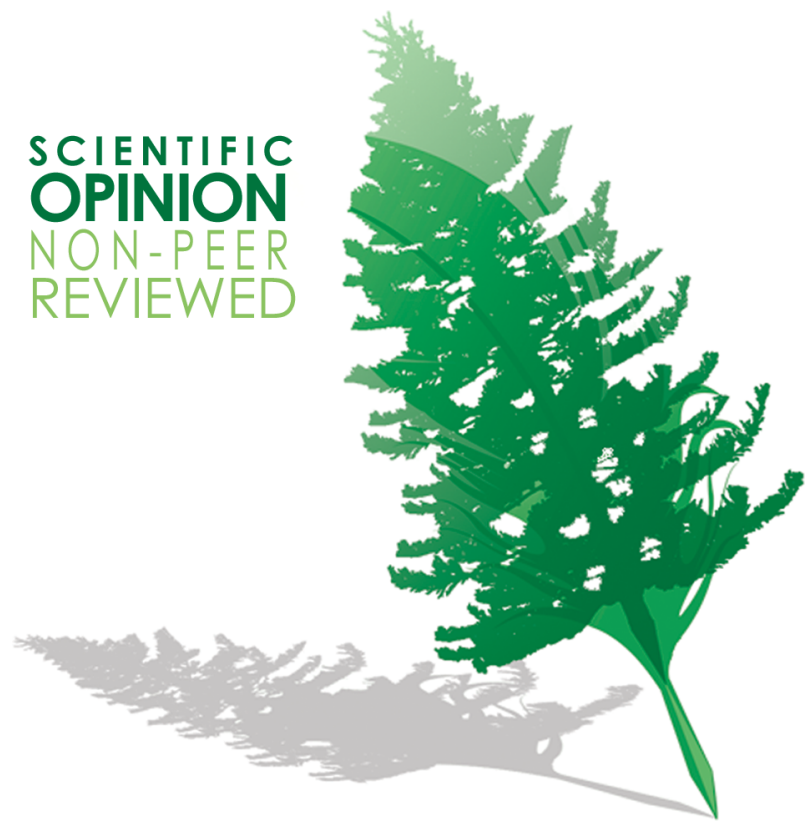

$\mathrm{M}$ ixtures of chlorinated paraffins (CPs) are widely used as additives in cutting fluids for metal working, as flame retardants in the rubber industry and as plasticizers in polyvinyl chloride production. ${ }^{1}$ In research studies, CPs are typically divided into short chain CPs (SCCPs), medium chain CPs (MCCPs), and long chain CPs (LCCPs), based on carbon chain length. The persistent organic pollutants review committee (POPRC) of the Stockholm Convention (SC) has been reviewing the draft profile of SCCPs since 2006. In May, 2017, at its eighth meeting, the Conference of Parties (COP8) of SC decided to list SCCPs in Annex A as a group of new POPs. ${ }^{2}$

The global production of CPs has increased sharply since the 1990s, especially in China. ${ }^{3}$ For example, the production and use of MCCPs has increased in recent years because MCCPs are the main components of $\mathrm{CP}-52$ products (the main $\mathrm{CP}$ commercial product in China). However, compared to SCCPs, data of environmental occurrence, transport pathways, and potential adverse effects to organisms for MCCPs is relatively scarce up to now. There are several reasons for this. First, researchers pay more attention to SCCPs since SCCPs have been proposed to be listed in the SC, while MCCPs have not. Second, there are big challenges in terms of the analytical method for MCCPs due to a) the mismatch of chlorination degree between the congener profiles of MCCPs standards and
MCCPs distributions in environmental samples, and b) mutual interferences of overlap in chromatograms and mass spectra between SCCPs and MCCPs using gas chromatography/liquid chromatography - mass spectrometer (GC/LC-MS) for instrument analysis, making peak resolution extremely difficult. Third, potential toxic interactions among SCCPs and MCCPs and the transformations between MCCPs and SCCPs in the environment have not been completely recognized.

Traditionally, the SC encourages their contracting parties to select appropriate alternatives to fill the application gap after the candidate POPs are restricted/eliminated by the Convention. However, many selected alternatives have later been proven to pose similar potential risks to the environment and human health since they usually are structurally similar to the candidate POPs. This reminds us the importance of studying relevant compounds (substances which can transform into each other or their derivatives with similar structures under certain conditions) from the very beginning. The POPRC reviewing process of polybrominated diphenyl ethers (PBDEs) is a vivid example of why we should pay more attention to substances that have similar structure. At its fourth meeting in May 2009, the Conference of the Parties (COP4) decided to add commercial penta- and octa-BDE products and their constituent congeners to the SC list of POPs. However, researchers had found that deca-bromodiphenyl ether (BDE 209) can be converted to lower bromine compounds in certain environmental or biological processes. For example, rainbow trout can biotransform BDE 209 primarily to octa- and nona-BDE congeners and even to hepta-BDE, ${ }^{4}$ which could provide an ongoing source over longer time periods to lower brominated PBDEs. Soon thereafter, the ninth of meeting of POPRC began to review the proposal to include deca-BDE and its mixtures in Annexes A, B and/or C of the SC in 2013. In May 2017, decaBDE was finally listed in Annex A with specific exemptions for the automotive and aerospace industries by COP8 of SC. ${ }^{2}$ Now, CP products are facing the similar situation. SCCPs have been listed in the SC to be controlled in 2017. It is expected that MCCPs will be chemicals of further concern of the environmental researchers and chemicals management in the future since they have been proven to have similar chemical structure, environmental behavior and toxicity to SCCPs. ${ }^{5}$

Chemicals with similar structure may have similar toxic effects and could transform into each other. For instance, perfluorooctanesulfonate (PFOS) and perfluorooctanoic acid

Received: June 26, 2017

Published: August 30, 2017 
(PFOA) show similar potencies as peroxisome proliferators in male rats. ${ }^{6}$ Meanwhile, tetrabromobisphenol A (TBBPA), is a neuro-toxin and emerging TBBPA derivatives have similar adverse effects, including neurotoxicity. For example, Liu et al. found that TBBPA derivatives have similar or even higher (compared to TBBPA) cellular toxicity to rat pheochromocytoma (PC12) cells. ${ }^{7}$ Likewise, while SCCPs and MCCPs share similar structures, they may also have similar nocuous characteristics. For example, the potential target organs of SCCPs and MCCPs both include liver, kidney, and thyroid. ${ }^{1}$

Despite different carbon chain length, SCCPs and MCCPs are all polychlorinated $n$-alkanes with similar physicochemical properties. They cannot be completely separated by chromatography and will generate characteristic ions with the same (when the $m / z$ value is presented with integer number) mass/ charge ratio. In addition, MCCPs and SCCPs are both important constituents in commercial CPs mixtures. This coexistence in CP products implied that SCCPs are difficult to be controlled without the regulatory control of MCCPs. On one hand, MCCPs might decompose into SCCPs through environmental chemical processes that result in breakdown of carbon-carbon bond or dechlorination. These processes include microbial degradation activities, wastewater treatment (ozone oxidation, chlorine disinfection, and ultraviolet radiation), and disposal of consumer goods that contain CPs (e.g., thermal treatment of scrap rubber/plastic). On the other hand, listing SCCPs in the SC will affect the CPs manufacturing industry, since manufacturers will need to improve technology to make sure their CPs products do not contain SCCPs (or limit the amount of SCCPs). Correspondingly, the MCCPs (as potential alternatives to SCCPs) fraction in CPs products are expected to increase worldwide, which implies that the emission of MCCPs to the environment will likely increase in the near future.

Therefore, there is urgent need to better identify the transformations, environmental fate, risk assessment of MCCPs, and interactions among SCCPs and MCCPs to the ecosystem and human health to tackle the issues that will result from phasing out of SCCPs. These are very important for the chemical managements for MCCPs in the future.

\section{AUTHOR INFORMATION}

\section{Corresponding Author}

*E-mail: ywwang@rcees.ac.cn.

\section{ORCID}

Yawei Wang: 0000-0002-6115-4076

Notes

The authors declare no competing financial interest.

\section{ACKNOWLEDGMENTS}

We greatly appreciated the support provided by the Chinese Academy of Sciences (XDB14010400) and the National Natural Science Foundation of China (21625702).

\section{REFERENCES}

(1) Fiedler, H. Short-chain chlorinated paraffins: production, use and international regulations. In: de Boer, J, editor. Chlorinated Paraffins. The Handbook of Environmental Chemistry; Springer-Verlag: Berlin Heidelberg, 2010.

(2) UNEP/POPS/COP.8/14, 2017, Recommendation by the Persistent Organic Pollutants Review Committee to list short-chain chlorinated paraffins in Annex A to the Convention and draft text of the proposed amendment.
(3) Wei, G. L.; Liang, X. L.; Li, D. Q.; Zhuo, M. N.; Zhang, S. Y.; Huang, Q. X.; Liao, Y. S.; Xie, Z. Y.; Guo, T. L.; Yuan, Z. J. Occurrence, fate and ecological risk of chlorinated paraffins in Asia: A review. Environ. Int. 2016, 92-92, 373-387.

(4) Stapleton, H. M.; Brazil, B.; Holbrook, R. D.; Mitchelmore, C. L.; Benedict, R.; Konstantinov, A.; Potter, D. In Vivo and In Vitro Debromination of Decabromodiphenyl Ether (BDE209) by Juvenile Rainbow Trout and Common Carp. Environ. Sci. Technol. 2006, 40, 4653-4658.

(5) UNEP/POPS/POPRC.6/11, 2010, Revised draft risk profile: short-chained chlorinated paraffins.

(6) Berthiaume, J.; Wallace, K. B. Perfluorooctanoate, perflourooctanesulfonate, and N-ethyl perfluorooctanesulfonamido ethanol; peroxisome proliferation and mitochondrial biogenesis. Toxicol. Lett. 2002, 129, 23-32.

(7) Liu, Q.; Ren, X. M.; Long, Y. M.; Hu, L. G.; Zhou, Q. F.; Jiang, G. $\mathrm{B}$. The potential neurotoxicity of emerging tetrabromobisphenol A derivatives based on rat pheochromocytoma cells. Chemosphere 2016, 154, 194-203. 